\title{
Atmosfera modificada na conservação da qualidade de uva 'Thompson Seedless' e na redução da podridão de Aspergillus
}

\author{
Rúbia Brito Camargo ${ }^{1}$, Daniel Terao ${ }^{2}$, Ana Rosa Peixoto ${ }^{3}$, Elizabeth Orika Ono ${ }^{4}$; Leonardo Souza Cavalcanti ${ }^{5}$; \\ Rodrigo Marques da Costa ${ }^{6}$
}

\begin{abstract}
${ }^{1}$ Engenheira agrônoma, mestranda em Horticultura Irrigada, Bolsista da FAPESB, Departamento de Tecnologia e Ciências Sociais, UNEB, Av. Edgard Chastinet, s/n, CEP: 48905-680, telefax: (074) 3611-5601, Juazeiro, BA. ${ }^{2}$ Dr. Pesquisador, Embrapa Semiárido. Petrolina/PE. ${ }^{3}$ Professor Titular, Departamento de Tecnologia e Ciências Sociais, UNEB. ${ }^{4}$ Professor Adjunto, Departamento de Botânica, Instituto de Biociências da Universidade Estadual Paulista, UNESP. ${ }^{5}$ Professor Adjunto do Colegiado de Engenharia Agrícola e Ambiental, Universidade do vale do São Francisco - UNIVASF, Juazeiro,BA. ${ }^{6}$ Graduado em Engenharia Agronômica, Departamento de Tecnologia e Ciências Sociais, UNEB.
\end{abstract} Autor para correspondência: Rúbia B. Camargo (biacamargo1@hotmail.com)

Data de chegada: 10/10/2010. Aceito para publicação em: 23/04/2012.

\section{RESUMO}

Camargo, R.B.; Terao D., Peixoto, A.R.; Ono, E.O.; Cavalcanti; L.S.; Costa, R.M. Atmosfera modificada na conservação da qualidade de uva 'Thompson Seedless' e na redução da podridão de Aspergillus. Summa Phytopathologica, v.38, n.3, p.216-222, 2012.

Existe uma demanda, na região semiárida produtora de uvas no Submédio São Francisco, por medidas sustentáveis de controle de doenças pós-colheita, uma vez que o modelo atual de revestimento de caixas com polietileno de alta densidade, associado ao metabissulfito de sódio, não tem se mostrado eficiente no controle dos fungos que ocorrem na região. O objetivo desse trabalho foi estudar um controle da podridão por Aspergillus em uvas 'Thompson Seedless' por meio da modificação da atmosfera, pelo envolvimento de caixas de uva em bolsões de poliamida. Comparou-se o bolsão de poliamida (PA) ao de polietileno alta densidade (PEAD), comumente usado na região, combinados ou não com o metabissulfito de sódio $\left(\mathrm{SO}_{2}\right)$. Frutos provenientes de propriedade comercial, após serem selecionados e desinfestados foram feridos com alfinete entomológico e inoculados com uma suspensão de Aspergillus niger na concentração de $10^{6}$ conídios.mL- ${ }^{1}$ e submetidos à câmara úmida por 24 horas. Em seguida as caixas de uva foram colocadas em bolsões específicos de acordo com o tratamento e armazenadas em câmara fria à temperatura de 2 ${ }^{\circ} \mathrm{C}$ e umidade relativa de $75 \%$, durante 40 dias. A partir do $12^{\circ}$ dia de armazenagem foram feitas avaliações semanais da incidência da doença e de variáveis físico-químicas: perda de massa, sólidos solúveis totais $(\mathrm{SST}), \mathrm{pH}$, acidez titulável $(\mathrm{AT})$, ratio $(\mathrm{SST} / \mathrm{AT})$; peroxidase (POD) e medição das concentrações de $\mathrm{CO}_{2}$ e $\mathrm{O}_{2}$ até o $40^{\circ}$ dia. $\mathrm{O}$ delineamento experimental utilizado foi inteiramente ao acaso em parcelas subdivididas com cinco repetições. O revestimento de caixas de uva em bolsões de poliamida, mesmo sem o uso de metabissulfito de sódio, apresenta-se como uma alternativa viável na manutenção da qualidade pós-colheita de uva "Thompson Seddless", bem como na redução de podridão causada por $A$. Niger. A enzima peroxidase pode ter atuado no processo de manutenção de qualidade da fruta, contribuindo para uma redução dos níveis da doença em uvas.

Palavras-chave adicionais: doenças pós-colheita, controle alternativo, poliamida.

\begin{abstract}
Camargo, R.B.; Terao D., Peixoto, A.R.; Ono, E.O.; Cavalcanti; L.S.; Costa, R.M. Modified atmosphere in preserving the quality of 'Thompson Seedless' grapes and reducing Aspergillus rot. Summa Phytopathologica, v.38, n.3, p.216-222, 2012.
\end{abstract}

There is a demand in Submédio São Francisco, a semiarid grape producing region in Brazil, for sustainable measures to control postharvest diseases, since the current model of coating boxes with high density polyethylene, associated with sodium metabisulfite, has not been effective in controlling fungi occurring in this region. The aim of this study was to evaluate the control of Aspergillus rot in 'Thompson Seedless' grapes by modifying the atmosphere, using polyamide bags to cover the grape boxes. Polyamide (PA) bags were compared with high density polyethylene (HDPE) bags, commonly used in this region, combined or not with sodium metabisulfite $\left(\mathrm{SO}_{2}\right)$. Fruits from a commercial area, after being selected and disinfested, were wounded with an entomological pin and inoculated with a suspension of Aspergillus niger at a concentration of $10^{6}$ conidia $\mathrm{mL}^{-}$ ${ }^{1}$ and then kept in a wet chamber for 24 hours. After that, grape boxes were placed in specific bags according to the treatment and stored at temperature of $2^{\circ} \mathrm{C}$ and relative humidity of $75 \%$ for 40 days. From the $12^{\text {th }}$ day of storage, the incidence of the disease and the physicochemical variables: mass loss, total soluble solids (TSS), $\mathrm{pH}$, titrable acidity (TA), ratio (TSS / TA); peroxidase (POD) and measurement of the concentrations of $\mathrm{CO}_{2}$ and $\mathrm{O}_{2}$ were weekly evaluated until the $40^{\text {th }}$ day. The experimental design was completely randomized in split plot with five replicates. The covering of grape boxes with polyamide bags, even without the use of sodium metabisulfite, showed to be a viable alternative for maintaining the postharvest quality of grapes 'Thompson Seddless', as well as for reducing rot caused by $A$. niger. The enzyme peroxidase may have acted in the process of maintaining fruit quality, contributing to a reduction in the level of the disease in grapes.

Additional keywords: postharvest disease, alternative control, polyamide. 
No Submédio do Vale do São Francisco o cultivo da videira (Vitis vinifera L.) tem relevante importância socioeconômica e vem se expandindo nos últimos anos, em virtude do incremento das técnicas de produção e demanda do mercado importador. Contudo, alguns aspectos relacionados à pós-colheita podem restringir a comercialização das uvas, nos casos em que é necessário o armazenamento por longo período, em virtude da queda na atividade fisiológica, da sensibilidade à desidratação e de infecções fúngicas (1).

As doenças fúngicas de pós-colheita ocorrem geralmente na forma de infecções quiescentes, adquiridas na fase de pré-colheita, quando os frutos ainda se encontram ligados às plantas. Porém, a partir do momento em que são colhidos, estes sofrem alterações fisiológicas tornando-se, mais susceptíveis ao ataque de patógenos $(3,6,21)$.

Pesquisas realizadas durante o período de 1994 - 1996 em amostras de uva 'Itália' (8) e em 2009 em amostras de uvas sem sementes na região do Submédio do Vale do São Francisco (4), constataram as presenças dos seguintes fungos: Aspergillus niger, Alternaria alternata, Cladosporium herbarum, Lasiodiplodia theobromae, Rhizopus stolonifer e Penicillium expansum, não sendo identificada a ocorrência do fungo Botrytis cinerea na região.

No entanto, provavelmente, devido ao desconhecimento dos agentes causais que provocam podridões em uva na região, o tratamento pós-colheita de uva, ainda está voltado para o controle do mofo cinzento, causado por $B$. cinerea, utilizando-se as tecnologias de regiões frias e úmidas, pela aplicação do anidrido sulfuroso $\left(\mathrm{SO}_{2}\right)$, na forma de fumigação ou gerado a partir do metabissulfito de potássio $\left(\mathrm{K}_{2} \mathrm{~S}_{2} \mathrm{O}_{5}\right)$ ou de sódio $\left(\mathrm{Na}_{2} \mathrm{~S}_{2} \mathrm{O}_{5}\right)$ no interior das embalagens que contêm os cachos $(5,12,16,24)$.

Assim, a eficiência do anidrido sulfuroso no controle dos fungos de maior ocorrência na região precisa ser comprovada, além desse produto poder provocar sintomas de fitotoxidez nas bagas e cachos, alterar o sabor das uvas e provocar reações alérgicas em pessoas sensíveis ao produto (2).

$\mathrm{O} A$. niger é a espécie fúngica mais frequente na região, principalmente quando a colheita ocorre em períodos chuvosos. A infecção inicia-se ainda no campo e os sintomas podem aparecer antes da colheita ou permanecer quiescente, manifestando-se gradativamente durante o período de armazenagem. Inicialmente ocorre um escurecimento e amolecimento do local infectado nas bagas, seguido de rompimento da casca, havendo no local, o desenvolvimento de um bolor escuro, que corresponde às estruturas de frutificação do fungo, que com o passar do tempo adquirem a uma tonalidade acinzentada, que poderá ser confundida com o mofo cinzento, inutilizando as bagas, resultando em grande prejuízo ao produtor.

Lichter et al. (17) relatam que o $\mathrm{SO}_{2}$ tem um largo espectro de ação contra vários microrganismos, mas sua atividade fungistática contra Aspergillus spp. ainda não foi comprovada.

Técnicas limpas e sustentáveis que visem o controle do A. niger, em associação com o resfriamento de frutas, estão sendo avaliadas com o intuito de garantir a segurança da saúde humana e o aumento da vida útil de prateleira do produto na pós-colheita e a minimização da incidência da podridão causada pelo fungo. Dentre essas técnicas, o uso de embalagens promotoras de modificação de atmosfera, como materiais a base de polietileno, polipropileno ou polipropileno orientado e poliamidas $(4,18)$ tem demonstrado resultados promissores. Os sistemas de modificação da atmosfera ocorrem pela redução da concentração de $\mathrm{O}_{2}$ e elevação da concentração de $\mathrm{CO}_{2}$, podendo desacelerar os processos fisiológicos prejudiciais à qualidade das uvas, bem como atuar indiretamente no controle de fungos pela ativação dos mecanismos de defesa fisiológica do fruto, impedindo o desenvolvimento dos fitopatógenos $(5,15,19,20,21)$.

Como existem poucas informações e recomendações na literatura relacionadas ao controle do $A$. niger, tanto no período de desenvolvimento do fruto, como em pós-colheita de uvas, este trabalho teve como objetivo estudar o efeito da atmosfera modificada como método alternativo para o controle da podridão pós-colheita causada por A. niger em uvas 'Thompson seedless'.

\section{MATERIAL E MÉTODOS}

\section{Preparo dos frutos e a inoculação}

Em outubro de 2009, $100 \mathrm{~kg}$ de uvas 'Thompson Seedless' foram coletados em uma propriedade particular, localizada em Juazeiro BA e enviados ao galpão de embalagem da propriedade, para limpeza e classificação de acordo com a qualidade estabelecida para o mercado europeu. No laboratório de Patologia Pós-colheita da Embrapa Semiárido, Petrolina-PE as uvas foram desembaladas, lavadas em água corrente por um minuto e posteriormente desinfestadas pela imersão em hipoclorito de sódio $1,5 \%$ durante 30 segundos e lavadas em seguida em água destilada estéril (ADE) e colocadas sobre papel absorvente. Depois os cachos foram pesados e acomodados em bandejas.

Os isolados de $A$. niger foram obtidos de baga de uva 'Itália' com sintomas de podridões provenientes de áreas comerciais do município de Petrolina-PE. Adicionou-se $10 \mathrm{~mL}$ de ADE sobre a superfície de colônias em crescimento ativo e a suspensão obtida foi filtrada com gaze dupla esterilizada e a contagem de esporos foi realizada em câmara de Neubauer, ajustando-se para uma concentração de esporos da ordem $10^{6}$ conídios $\mathrm{mL}^{-1}$.

Em cada cacho de uva, marcaram-se dez bagas onde foram realizados ferimentos com alfinete entomológico a uma profundidade aproximada de 2,0 $\mathrm{mm}$. Sobre o ferimento de cada baga foi aplicado a suspensão do inóculo utilizando-se pulverizador manual. Após a inoculação, os cachos foram dispostos em caixas plásticas com tampas e mantidos em câmara úmida por 24 horas, sendo então transferidos para câmara fria a $2^{\circ} \mathrm{C}$ e UR a $75 \%$.

\section{Aplicação dos tratamentos}

Os cachos inoculados foram acondicionados em caixas de papelão, que foram envolvidas em bolsões específicos e submetidos aos seguintes tratamentos: T1 - poliamida (PA) sem metabissulfito de sódio; T2 - poliamida (PA) com metabissulfito de sódio; T3 polietileno alta densidade (PEAD) sem metabissulfito de sódio; T4 polietileno alta densidade (PEAD) com metabissulfito de sódio. Os tratamentos T3 e T4 serviram como testemunhas, uma vez que utilizaram os bolsões comumente usados na região, associado ou não ao metabissulfito de sódio. Para cada tratamento, foram usadas cinco repetições, sendo que cada repetição era constituída de dois cachos de uva contidos em um recipiente de plástico rígido transparente. A armazenagem foi feita em câmara fria à temperatura de $2{ }^{\circ} \mathrm{C}$ e a umidade relativa de 75 durante 40 dias.

Após 40 dias de armazenagem realizaram-se as avaliações fitopatológicas, pela incidência da doença e físico-químicas: perda de massa, $\mathrm{pH}$, teor de sólidos solúveis totais (SST), acidez titulável (AT), ratio (SST/AT) e análise de peroxidase (POD) e leitura da concentração dos gases $\left(\mathrm{CO}_{2} / \mathrm{O}_{2}\right)$. A perda de massa foi avaliada realizando-se a pesagem dos cachos individualmente, em balança de precisão. $\mathrm{O}$ pH, foi obtido utilizando-se pHmetro digital, modelo NT pH 2; o teor de SST foi obtido por meio de refratômetro digital; a AT foi determinada 
por titulação com solução de $\mathrm{NaOH} 0,1 \mathrm{~N}$. A atividade da POD foi determinada adaptando-se o método utilizado por Clemente (11): após a mistura da solução de $2,75 \mathrm{~mL}$ de peróxido de hidrogênio a $10 \%$ e da solução tampão acetato sódio de anidro $(50 \mathrm{mM}$ e pH 5,5$)$, foi adicionado $1 \mathrm{~mL}$ do extrato vegetal e finalmente $0,16 \mathrm{~mL}$ de guaiacol a $1 \%$. O resultado dessa mistura foi incubado a $30^{\circ} \mathrm{C}$ por 20 minutos e as leituras foram realizadas em espectrofotômetro Shimatzu, programa UV Probe a $480 \mathrm{~nm}$.

As leituras das concentrações de $\mathrm{CO}_{2} / \mathrm{O}_{2}$, foram realizadas com aparelho succionador desses gases, onde uma agulha de $20 \mathrm{G} \mathrm{e} 0,9 \mathrm{~mm}$ de diâmetro era inserida na embalagem e a sucção dos gases era realizada durante um minuto. Após a retirada da agulha orifício era lacrado com fita adesiva, para evitar a perda dos gases. $\mathrm{O}$ aparelho utlizado foi o CheckPoint, PBI Dansensor.

Os materiais plásticos usados foram: poliamida LifeSpan ${ }^{\circledR}, \operatorname{cod}$. L 605, constituída de polímeros de cadeia linear, em bolsões microperfurados com $30 \mu \mathrm{m}$ de espessura e o polietileno alta densidade constituída de polímeros de cadeia ramificada, em bolsões microperfurados com $20 \mu \mathrm{m}$ de espessura e uma taxa de ventilação entre 0,5 a $2,0 \%$.

O delineamento experimental utilizado foi inteiramente casualizado, em parcelas subdivididas, com cinco repetições. $\mathrm{Na}$ análise de variância dos dados (ANOVA) utilizou-se o programa ASSISTAT e as médias dos tratamentos foram comparadas pelo Teste de Tukey a $1 \%$ de probabilidade e pelo teste de Scott-Knott para análise de peroxidase.

\section{RESULTADOS E DISCUSSÃO}

Observa-se pela Figura 1, que em todas as avaliações, a incidência da doença foi menos expressiva no tratamento em que as caixas com uvas foram envolvidas em sacos de poliamida microperfurada associado ao metabissulfito de sódio (T2), diferindo significativamente dos tratamentos com polietileno de alta densidade (T3 e T4), não diferindo do tratamento com poliamida sem metabissulfito de sódio (T1) aos 40 dias de armazenagem.

Neves et al. (20) quando avaliaram a conservação pós-colheita das uvas 'Crimson Seedless' e 'Itália' submetidas a diferentes tipos de embalagens e a ação do $\mathrm{SO}_{2}$, verificaram que, em ambas as variedades, as uvas submetidas à ação do gerador de $\mathrm{SO}_{2}$, contendo $3 \mathrm{~g}$ de metabissulfito de sódio e acondicionamento em embalagens de polietileno de baixa densidade (PEBD) de 0,02 $\mathrm{mm}$ de espessura, apresentaram menor percentual de bagas deterioradas.

Constata-se pela Figura 2 e 3, que aos 40 dias de armazenagem, quando foi possível observar, com melhor evidência, os resultados favoráveis do uso da embalagem da poliamida associado ao metabissulfito de sódio no controle da podridão causada por $A$. niger, uma elevação considerável na concentração de $\mathrm{CO}_{2}$ ultrapassando a concentração de $\mathrm{O}_{2}$. De acordo com Sommer (26) uma das vantagens na utilização de sistemas com promoção da modificação de atmosfera no interior da embalagem, a exemplo do PEBD (polietileno baixa densidade), é que poderá contribuir para o retardamento no surgimento dos sintomas da doença em decorrência tanto do atraso na senescência como de um efeito direto de voláteis sobre o desenvolvimento dos fungos. A utilização de embalagens plásticas com materiais de baixa densidade tem-se mostrado, também, como uma alternativa eficiente para preservação da qualidade por período de tempo superior em relação ao polietileno de alta densidade (PEAD), além de servir como uma barreira física, contribuindo para redução de possíveis contaminações $(9,14)$.

Comparando-se as medições realizadas de concentração de $\mathrm{CO}_{2} \mathrm{e}$ de $\mathrm{O}_{2}$ na ausência de metabissulfito de sódio (Figura 02) e na presença (Figura 03) observou-se resultados similares aos obtidos por Pretel et al. (22), que estudando a qualidade da colheita tardia em uvas de mesa 'Aledo' em ambiente refrigerado, seguido por um período de 4 dias a $20^{\circ} \mathrm{C}$ que foram submetidos a quatro tratamentos: controle sem $\mathrm{SO}_{2}$; atmosfera ligeiramente enriquecida com $\mathrm{CO}_{2}$ versus $\mathrm{SO}_{2}$; controle com $\mathrm{SO}_{2}$ e atmosfera ligeiramente enriquecida com $\mathrm{CO}_{2}$ versus $\mathrm{SO}_{2}$,

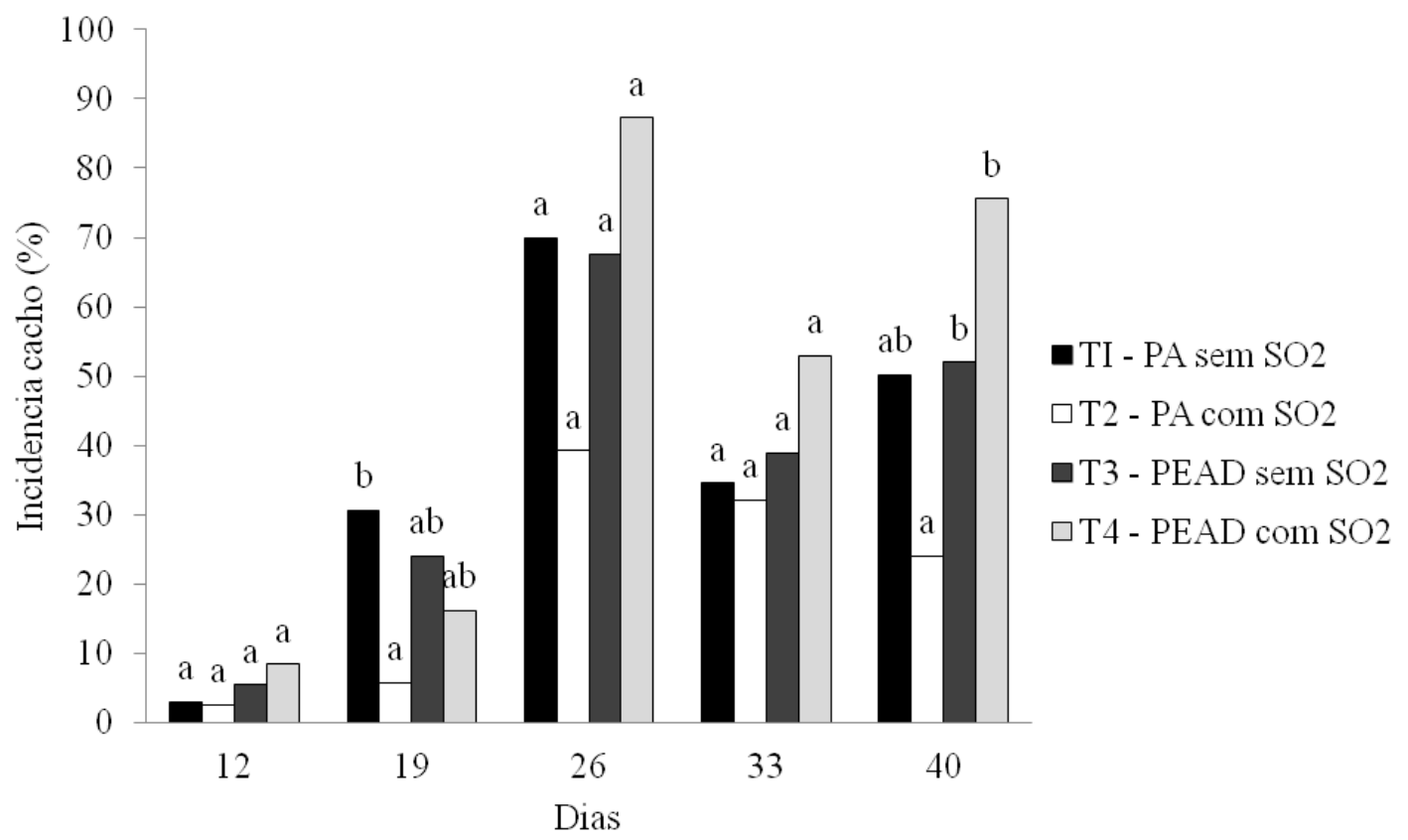

Figura 1. Incidência de Aspergillus niger em uvas 'Thompson Seedless' inoculadas, submetidas aos tratamentos com poliamida (PA) e polietileno alta densidade (PEAD) combinados ou não com o metabissulfito de sódio $\left(\mathrm{SO}_{2}\right)$ e armazenadas em câmara fria à temperatura de $2{ }^{\circ} \mathrm{C}$ e umidade relativa de 75 $\%$ durante 40 dias.

Médias seguidas de mesma letra não diferem entre si pelo teste de Tukey ao nível de $1 \%$ de probabilidade. 


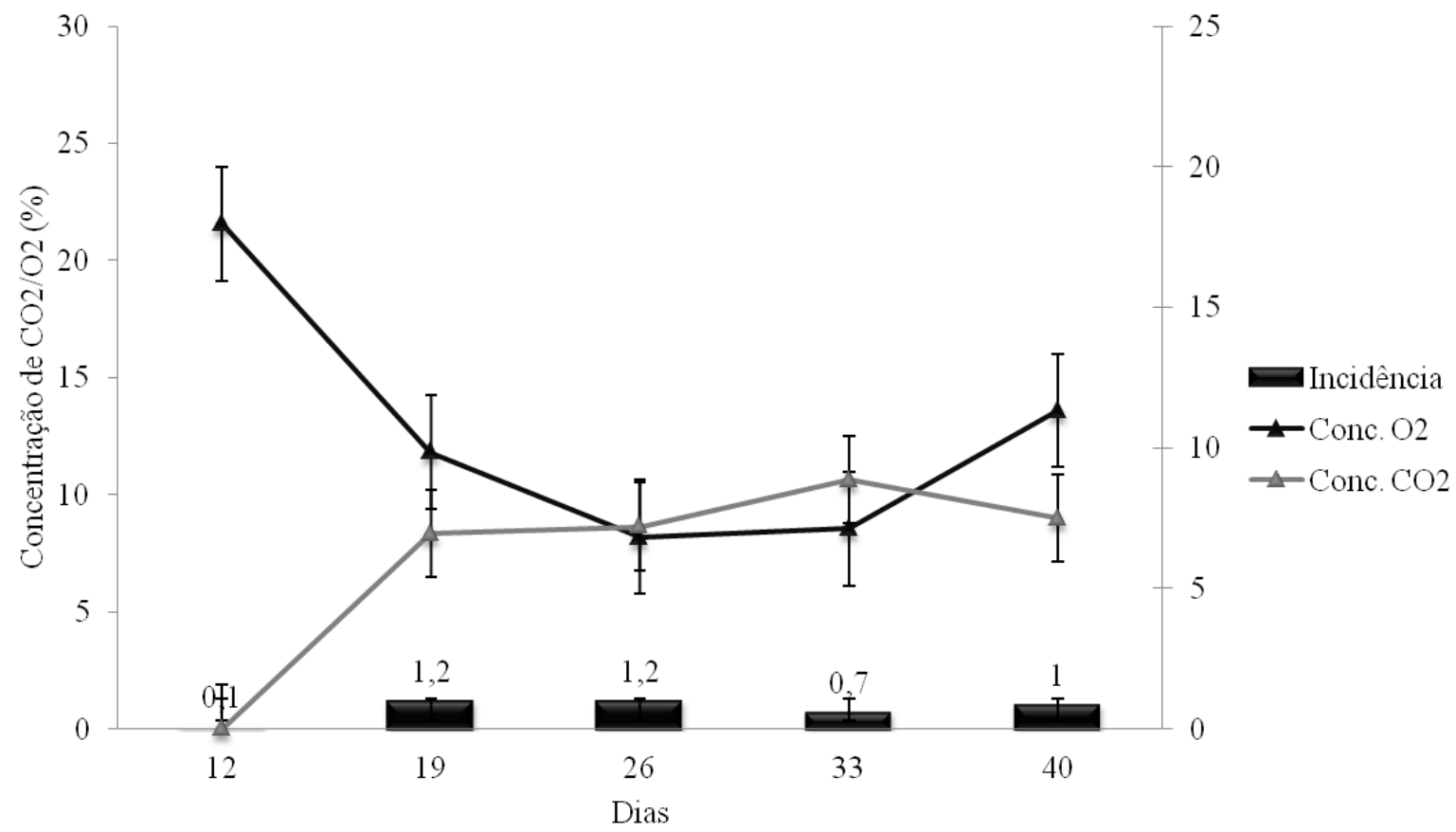

Figura 2. Incidência de Aspergillus niger e concentrações de $\mathrm{CO}_{2} / \mathrm{O}_{2}$ em ambiente de atmosfera modificada (Bolsão de poliamida sem metabissulfito de sódio) em um período de 40 dias de armazenagem, em câmara fria à temperatura de $2{ }^{\circ} \mathrm{C}$ e umidade relativa de $75 \%$, utilizando-se cinco repetições.

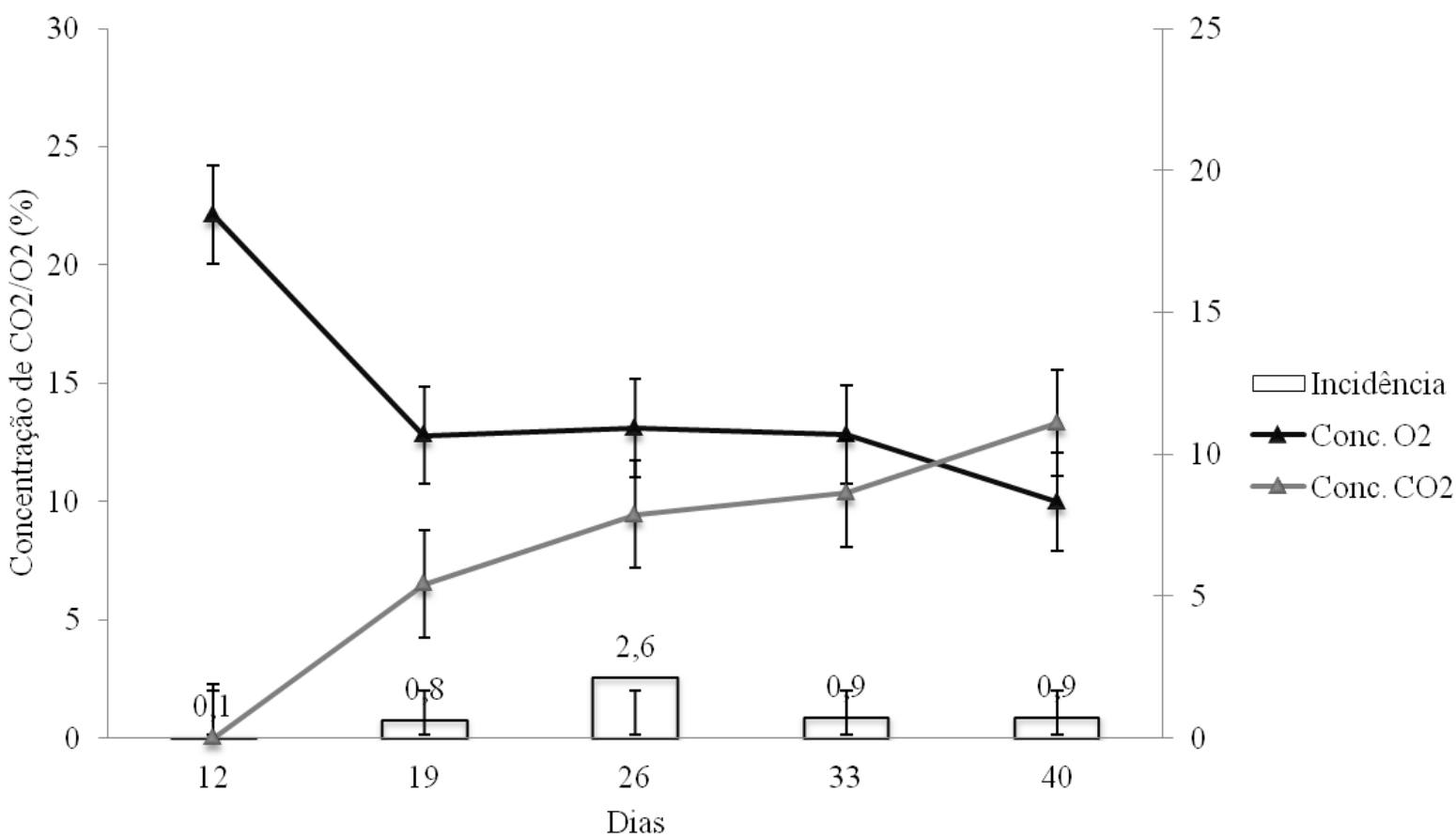

Figura 3. Incidência de Aspergillus niger e concentrações de $\mathrm{CO}_{2} / \mathrm{O}_{2}$ em ambiente de atmosfera modificada (Bolsão de poliamida com metabissulfito de sódio) em um período de 40 dias de armazenagem, em câmara fria à temperatura de $2{ }^{\circ} \mathrm{C}$ e umidade relativa de $75 \%$, utilizando-se cinco repetições.

concluíram que a combinação da atmosfera ligeiramente enriquecida com $\mathrm{CO}_{2}$ versus $\mathrm{SO}_{2}$, teve um efeito sinérgico sobre as uvas, reduzindo as taxas proliferação de $B$. cinerea.

Prusky et al. (23) relataram que as concentrações de $\mathrm{O}_{2}$ e $\mathrm{CO}_{2}$ requeridas para inibir o desenvolvimento e a germinação de esporos variam com a espécie do fungo. Cia (10), quando avaliou o efeito da atmosfera modificada (AM) no controle de podridões pós-colheita e no aumento de vida útil do caqui 'Fuyu' em ambiente refrigerado utilizando: embalagens de PVC $12 \mu \mathrm{m}$ (policloreto de vinila); PEBD $80 \mu \mathrm{m}$ (polietileno de baixa densidade); PEBD $80 \mu \mathrm{m}\left(3 \% \mathrm{O}_{2} / 8 \%\right.$ $\mathrm{CO}_{2}$ ); PO $63 \mu \mathrm{m}$ (multicamada coextrusado à base de poliolefinas); PO $63 \mu \mathrm{m}\left(3 \% \mathrm{O}_{2} / 8 \% \mathrm{CO}_{2}\right)$; PO $58 \mu \mathrm{m}$; PEBD $50 \mu \mathrm{m}$; PO $38 \mu \mathrm{m}$ microperfurado; PEBD $50 \mu \mathrm{m}\left(3 \% \mathrm{O}_{2} / 8 \% \mathrm{CO}_{2}\right)$; PEBD $50 \mu \mathrm{m}(3 \%$ $\mathrm{O}_{2} / 10 \% \mathrm{CO}_{2}$ ), concluiu que o filme com a espessura de $50 \mu \mathrm{m}$, apresentou excelentes resultados para o armazenamento de caqui, inibindo o desenvolvimento de R. stolonifer (Ehrenb) Vuill, mantendo 
a qualidade dos frutos por 84 dias. Entretanto, observou que a injeção de mistura gasosa não teve efeito no aumento do período de armazenamento dos frutos.

Quanto às variáveis físico-químicas, para perda de peso até o $33^{\circ}$ dia não houve diferença significativa entre os tratamentos (Tabela 1). Entretanto, ao $40^{\circ}$ dia observa-se diferença significativa dos tratamentos com poliamida microperfurada (T1) e polietileno de alta densidade (T3 e T4) apresentando aproximadamente, uma diferença de peso de $45 \%$, demonstrando haver uma tendência para redução na perda de peso da uva, para armazenamento em longo prazo, pela utilização de bolsão de poliamida, em relação ao bolsão de polietileno de alta densidade, comumente usado pelos produtores da região. Esses dados estão em conformidade aos encontrados por Yamashita et al. (27), em pesquisa desenvolvida com uva 'Itália', submetidas a três diferentes filmes promotores de modificação de atmosfera (AM), constataram que para tratamentos com AM, houve uma perda de massa 28 vezes menor em relação as uvas não submetidas a modificação de atmosfera, prolongando a vida útil da uva em até 63 dias.

Martínez-Romero et al. (19), em pesquisas feitas com uvas 'Flame Seedless' concluíram que quando estas foram submetidas ao tratamento com polipropileno não orientado, aos 53 dias de armazenada, tiveram maior concentração de $\mathrm{CO}_{2}$ e menor de $\mathrm{O}_{2}$, contribuindo para uma menor perda de peso e firmeza de bagas. Neves et al. (20), também comprovaram em trabalhos realizados com uvas "Crimson Seedless' e Itália, submetidas ao tratamento com $\mathrm{SO}_{2}$ e associadas ao PEBD, independente o tipo de embalagem secundária, apresentaram, até os 56 dias, menor perda de massa fresca, menor taxa de degrana e de bagas deterioradas em função da manutenção da qualidade dos frutos.

Para as variáveis físico-químicas (Tabela 2) de modo geral, percebese que as embalagens não exerceram influência quanto ao teor de SST, $\mathrm{pH}$ e ratio (SST/AT). Este dado é interessante em termos qualitativos, pois demonstra que o tipo de embalagem não é fator determinante para manutenção dessas características. Diversos pesquisadores
$(2,12,19,20,27)$ afirmaram que o tipo de embalagem utilizados para pesquisas realizadas em uvas, não influenciou nos teores de $\mathrm{pH}$; SST e AT. Entretanto, quando se avaliou a relação final ratio (SST/AT), houve diferença significativa entre os tratamentos, sendo que os tratamentos com poliamida apresentaram os melhores resultados. Também, observou-se no tratamento com PEAD com metabissulfito um incremento do ratio, enquanto para os demais houve redução (Tabelas 1 e 2). Segundo Martínez-Romero et. al (19), este fato está associado a perda de peso das uvas, favorecendo uma elevação das concentrações de sólidos solúveis totais.

Com relação à atividade da enzima peroxidase (POD), observouse que no T1 (Poliamida sem metabissulfito) ocorreu um crescimento linear a partir do $19^{\circ}$ dia, atingindo um pico de atividade aos 33 dias depois de armazenada, apresentando valores superiores aos demais tratamentos (Figura 4). Já para o T2 (poliamida com metabissulfito) houve um pico, ainda que reduzido da POD aos 19 dias. Para os tratamentos com PEAD (T3 e T4) não houve diferenças significativas durante o período de avaliação, havendo menor atividade enzimática. Segundo Chitarra \& Chitarra (7) a atividade da POD aumenta significativamente após a colheita, quando uma gama de compostos torna-se susceptível à sua ação e exemplifica essa atividade em maçã, onde o pico da POD solúvel ocorre na metade do processo de amaciamento relacionado ao amadurecimento, independente das condições de armazenamento e da taxa de amaciamento.

Edagi et al. (13), em pesquisa realizada com nêsperas 'Fukuhara' com o objetivo de avaliarem a eficiência de tratamentos térmicos no aumento do potencial de frigoconservação e os efeitos desses tratamentos na qualidade e nas propriedades físico-químicas e bioquímicas dos frutos de nêsperas, concluíram que os tratamentos térmicos não evitaram o enrijencimento de polpa, e que durante o armazenamento refrigerado, foi observado aumento da firmeza de polpa para os tratamentos realizados, causado pela lignificação do tecido, catalisada principalmente pelas enzimas fenilalanina amônioliase (PAL)

Tabela 1. Efeito da perda de massa (g) em uvas 'Thompson Seedless' sob dois tipos de embalagens: poliamida (PA) e polietileno de alta densidade (PEAD), submetidas ou não ao metabissulfito de Sódio $\left(\mathrm{SO}_{2}\right)$, após $12,19,26,33$ e 40 dias de armazenagem em câmara fria a $2^{\circ} \mathrm{C}$ e UR $75 \%$.

\begin{tabular}{lllccc}
\hline Tratamentos & \multicolumn{3}{c}{ Dias (perda de massa - g) } & $\mathbf{3 3}^{\mathbf{0}}$ & $\mathbf{4 0}^{\mathbf{0}}$ \\
\cline { 2 - 5 } & $\mathbf{1 2}^{\mathbf{0}}$ & $\mathbf{1 9}^{\mathbf{0}}$ & 8,395 & 10,627 & $5,951 \mathrm{c}$ \\
T1 - PA sem SO & 3,906 & 5,756 & 9,630 & 6,975 & $7,510 \mathrm{bc}$ \\
T2 - PA com SO & 6,174 & 7,887 & 11,437 & 10,480 & $13,304 \mathrm{a}$ \\
T3 - PEAD sem SO & 6,461 & 10,303 & 10,188 & 12,377 & $11,685 \mathrm{ab}$ \\
T4 - PEAD com SO SO $_{2}$ & 6,848 & 9,055 & & 64,45 \\
CV(\%) & & & & \\
\hline
\end{tabular}

Médias seguidas de mesma letra não diferem entre si ao nível de $1 \%$ de probabilidade, pelo teste de Tukey.

Tabela 2. Avaliação de variáveis físico-químicos: pH, SST e a relação SST/AT em uvas 'Thompson Seedless' sob dois tipos de embalagens: poliamida (PA) e polietileno de alta densidade (PEAD), submetidas ou não ao metabissulfito de Sódio $\left(\mathrm{SO}_{2}\right)$, após 12, 19, 26,33 e 40 dias de armazenagem em câmara fria a $2^{\circ} \mathrm{C}$ e UR $75 \%$.

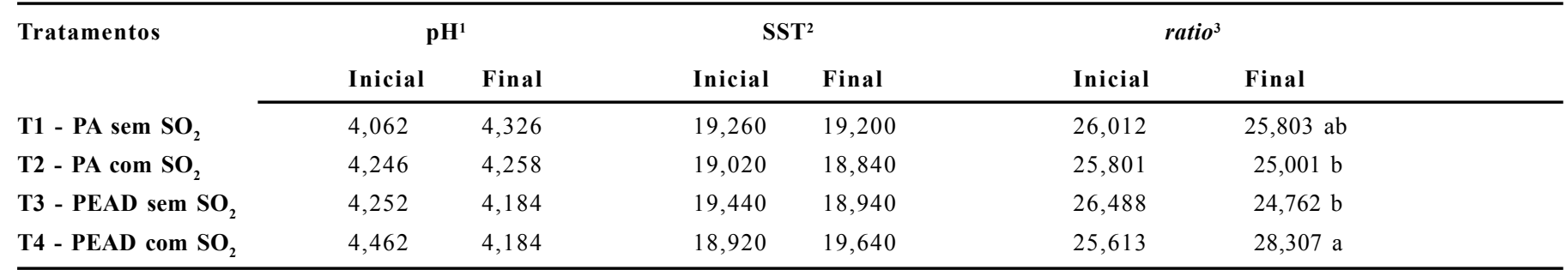

Médias seguidas de mesma letra não diferem entre si pelo teste de Tukey ao nível de $1 \%$ de probabilidade. 


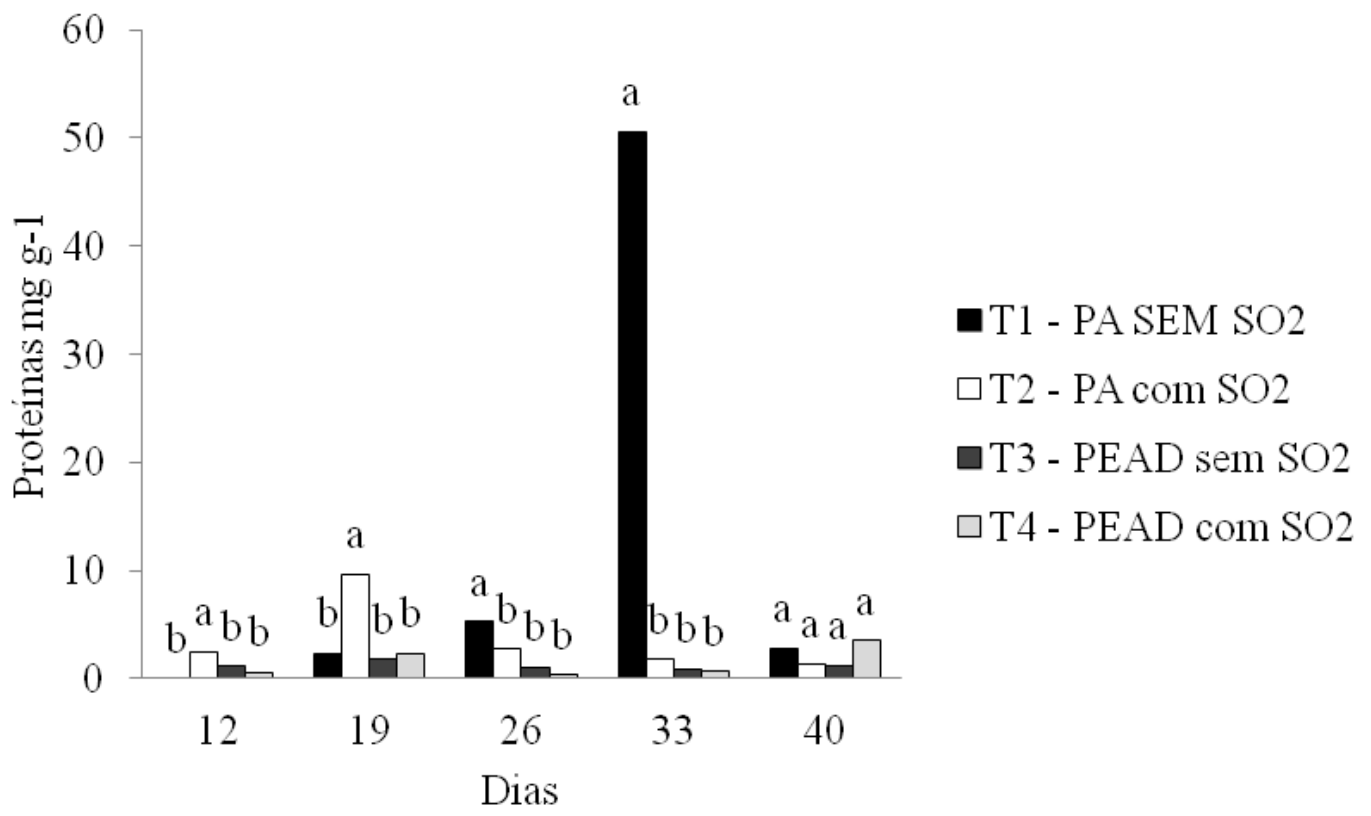

Figura 4. Atividade da enzima peroxidase em uvas sob dois tipos de embalagens de polietileno: poliamida (PA) e polietileno alta densidade (PEAD) combinados ou não com o metabissulfito de sódio $\left(\mathrm{SO}_{2}\right)$ e armazenadas em câmara fria à temperatura de $2{ }^{\circ} \mathrm{C}$ e umidade relativa de 75 \% durante 40 dias. Médias seguidas das mesmas letras não diferem entre si ao nível de $1 \%$ de probabilidade, pelo teste de Scott-Knott.

e peroxidase (POD). Da maneira similar, Singh \& Dwivedi (25) afirmaram que a POD estaria relacionada à polimerização de monômeros de lignina. Esse fato pode influenciado na menor incidência da doença nos frutos em caixas ensacadas em bolsões de poliamida, bem como na diminuição da perda de peso durante o período de armazenagem (Tabela 1), tendo como consequência uma melhor aparência visual e um aumento de vida útil pós-colheita dos frutos.

\section{CONCLUSÃO}

O revestimento de caixas de uva em embalagens de poliamida, mesmo sem o uso de metabissulfito de sódio, apresenta-se como uma alternativa viável na manutenção da qualidade pós-colheita de uva "Thompson Seddless" bem como na redução de podridão causada por Aspergillus niger.

\section{REFERÊNCIAS BIBLIOGRÁFICAS}

1. Artés-Hernàndez, F.; Tomás-Barberán, F. A. Modified atmosphere packaging preserves quality of $\mathrm{SO}_{2}$-free 'Superior seedless' table grapes. Postharvest Biology and technology, Amsterdan, v. 39, p. 146-154. 2006.

2. Artés-hernàndez, F.; F. Artéz, E. Aguayo. Alternative atmosphere treatments for keeping quality of 'Autumn seedless' table grapes during long-term cold store. Postharvest Biology and technology, Amsterdan, v. 31, p. 56-67. 2004.

3. Barkai-Golan, R. Postharvest diseases of fruits and vegetables: development and control. Amsterdan: Elsevier Science, 2001. 418 p.

4. Camargo, R. B.; Peixoto, A. R.; Terao, D.; Ono, E. O.; Cavalcanti, L. S. Fungos causadores de podridões pós-colheita em uvas apirênicas no pólo agrícola de Juazeiro-BA e Petrolina-PE. Revista Caatinga, Mossoró, v. 24, n. 1, p. 15-19, 2011.

5. Camili, E. C et al. Avaliação de quitosana, aplicada em pós-colhei- ta, na proteção de uva 'Itália' contra Botrytis cinérea. Summa Phytopathology, Botucatu, v. 33, p.3, p. 215-221, 2007.

6. Cavalcanti, L.S.; Di Peiro, R.M.; Cia, P.; Pascholati, S.F.; Resende, M.L.V.; Romeiro, R.S. Indução de resistência em plantas a patógenos e insetos. Piracicaba: FEALQ, 2005. 263 p.

7. Chitarra, M. I. F; Chitarra, A. B. Pós-colheita de frutas e hortaliças: fisiologia e manuseio. 2. ed., Lavras: UFLA, 2005. 785 p.

8. Choudhury, M.M. Fungos associados à deterioração patológica póscolheita em uva de mesa (cv. Itália) produzida no submédio São Francisco, Sociedade Brasileira de Fruticultura, Curitiba, v. 14, p. 400, 1996. In: Congresso Brasileiro de Fruticultura, Curitiba, v. 14, p. 400, 1996 (Resumo)

9. Cia, P. Efeito de atmosfera modificada no controle de podridóes pós-colheita e na qualidade de caqui cv. Fuyu. 2002. 132 p. Dissertação (Mestrado em Agronomia) - Faculdade de Ciências Agronômicas, Universidade Estadual Paulista "Júlio de Mesquita Filho", Botucatu.

10. Cia, P.; Bron, I. R.; Valentini, S. R. T.; Pio, R.; Chagas, E. A. Atmosfera modificada e refrigeração para conservação pós-colheita da amora-preta. Bioscience Journal, Uberlândia, v. 23, n. 3, p. 11-16, 2007.

11. Clemente, E.; Pastore, G. M. Peroxidase and polyphenoloxidase, the importance for food technology. Ciência e Tecnologia de Alimentos, Campinas, v. 32, n. 2, p. 167- 171, 1998.

12. Crisosto, C. H.; Garner, D.; Crisosto, G. Carbon dioxide-enriched atmospheres during cold storage limit losses from Botrytis but accelerate rachis browning of 'Redglobe' table grapes. Postharvest Biology and Tecnology, Amsterdan, v. 26, p. 181-189, 2002 .

13. Edagi, F. K.; Sestari, I; Sasaki, F. F.; Cabral, S. M.; Meneghini, J.; Kluge, R. A. Aumento do potencial de armazenamento refrigerado de nêsperas 'Fukuhara' com o uso de tratamento térmico. Pesquisa Agropecuária Brasileira, Brasília, DF, v.44, p. 1270 - 1276, 2009.

14. Ferri, V. C; Rinaldi, M. M.; Danieli, R.; Lucchetta, L. Rombaldi, C. V. Atmosfera modificada na conservação de caquis (Diospyrus $k a k i$, L) cultival Fuyu. Revista Brasileira de Agrociência, Pelotas, v. 10, n. 1, p. 111-115, 2004. 
15. Floros, J.; Matsos, K. I. Introducion to modified atmosphere packaging. Inovations in food packaging. London, Elsevier, 2005, p. 159-172.

16. Kugle, R. A. et al. Fisiologia e manejo pós-colheita de frutas de clima temperado. Campinas, Livraria e Editora Rural, 2002, $214 \mathrm{p}$.

17. Lichter, A.; Zutkhy, Y.; Sonego, L.; Dvir, O.; Kaplunov, T.; Sarig, P.; Ben-Arie, R. Ethanol controls postharvest decay of table grapes. Postharvest Biology and Technology, Amsterdan, v. 24, p. 301-308. 2002

18. Lima, M. A. C.; Alves, R. E.; Assis, J. S.; Filgueiras, H. A. C.; Costa, J. A. T. Aparência, compostos fenólicos e enzimas oxidativas em uva 'Itália' sob influência do cálcio e do armazenamento refrigerado. Revista Brasileira de Fruticultura. Jaboticabal, v. 24, n. 1, p. 39-43, 2002.

19. Martinez-Romero, D. M.; Guillén, F.; Castillo, S; Valero, D.; Serrano, M. Modified atmosphere packaging maintains quality of table grapes. Journal Food Science, Malden, v. 68, n.5, 2003.

20. Neves, L.C; Silva, V.X; Benedette, R.M. Conservação de uvas "Crimson seedless" e "Itália", submetidas a diferentes tipos de embalagens e dióxido de enxofre $\left(\mathrm{SO}_{2}\right)$. Revista Brasileira de Fruticultura, Jaboticabal, v.30, n.1, p.65 - 73, 2008.

21. Oliveira, S. M. A; Terao, D.; Dantas, S. A. F.; Tavares, S. C. C. H.. Patologia pós-colheita. In: Oliveira, S. M. A; Terao, D.; Dantas, S. A. F.; Tavares, S. C. C. H. Patologia pós-colheita: frutas, olerícolas e ornamentais tropicais. Brasília, DF: Embrapa Infor- mação Tecnológica, 2006, 855 p.

22. Pretel, M. T.; Martínez-Madrid, M. C.; Martínez, J. R.; Carreño, J. C.; Romojaro, F. Prolonged storage of 'Aledo' table grapes in a slightly $\mathrm{CO} 2$ enriched atmosphere in combination with generators of $\mathrm{SO}_{2}$. Lebensmittel-Wissenschaft und Technologie, London, v. 39, p. 1109-1116, 2006.

23. Prusky, D.; Perez, A.; Zutkhi, Y.; Ben-Arie, R. Effect of modified atmosphere for control of black spot, caused by Alternaria alternata, on stored persimmon fruits. Annual Review Phytopathology, Palo Alto, v.87, p. 203-208, 1997.

24. Retamales, J; Defilippi, B. G.; Arias, M.; Castilho, P.; Marínquez, D. High- $\mathrm{CO}_{2}$ controlled atmospheres reduce decay incidence in Thompson Seedless and Red Globe table grapes. Postharvest Biology and Technology, Amsterdan, v 29, p. 177-/182, 2003.

25. Singh, R.; Dwivedi, U.N. Effect of ethrel and 1methylcyclopropene (1MCP) on antioxidants in mango (Mangifera indica var. Dashehari) during fruit ripening. Food Chemistry, Barking, v.111, p.951956, 2008.

26. Sommer, N. Role of controlled environments in suppression of postharvest diseases. Canadian Journal Plant Pathology, Peterborough, v.7, p. 331-339, 1985

27. Yamashita, F.; Tonzar, A. C.; Fernandes, J. G.; Moriya, S.; Benassi, M. T. Influência de diferentes embalagens de atmosfera modificada sobre a aceitação de uvas de mesa var. Itália mantidas sob refrigeração. Ciência e Tecnologia de Alimentos, Campinas, v. 20, n.1, p. 110-114, 2000. 